\title{
COVID CONCEPTS
}

\section{Maskne: Exacerbation or Eruption of Acne During the COVID-19 Pandemic}

\author{
Tamar Aliya Gomolin $\mathrm{BSc}^{1}$, Abigail Cline, $\mathrm{PhD}, \mathrm{MD}^{2}$, Marian Russo, $\mathrm{MD}^{2}$ \\ ${ }^{1}$ New York Medical College, Valhalla, NY \\ ${ }^{2}$ Department of Dermatology, Metropolitan Hospital, New York, NY
}

\section{ABSTRACT}

Although the COVID-19 outbreak has decreased dermatology outpatient clinic visits, acne remains the most common condition among patients requesting an appointment. The widespread use of face masks may be a contributing factor to acne mechanica and folliculitis mechanica, where stopping the mechanical insult is essential to treatment. However, COVID-19 poses a unique challenge as mask wearing is crucial to limiting viral exposure. Although reported cases of mask-associated facial dermatoses are largely documented in healthcare workers, the general population is being affected by "maskne". Pathogenesis and treatment options are discussed and the importance of counseling patients on proper skin hygiene is highlighted. As mask use increases, dermatologists should anticipate this trend in acne flare-ups.

Although COVID-19 has decreased dermatology outpatient clinic visits, acne remains the most common condition among patients requesting appointments. ${ }^{1}$ The hashtag "maskne" is trending on Instagram with over 22,000 posts detailing this widespread struggle.

The use of masks may be a contributing factor to acne mechanica. ${ }^{1}$ Acne mechanica is unique from acne vulgaris which includes symptoms like burning and/or pruritus. ${ }^{2}$ Additionally, pruritus induces scratching, thereby aggravating acne mechanica and compromising mask protection. Stopping mechanical insults is crucial to treating acne mechanica; ${ }^{2}$ however, COVID-19 poses a unique challenge as mask wearing is crucial to limiting exposure.

Besides mechanical factors, mask wearing exacerbates acne due to sweating and increased humidity, leading to swelling of epidermal keratinocytes of the pilosebaceous follicle and obstruction. ${ }^{3}$ Changes to surface sebum composition and skin hydration may disrupt the skin barrier, leading to changes in skin microflora. ${ }^{3}$ Therefore, "maskne" treatment should center on maintaining skin barrier integrity.

Dermatologists have proposed the term folliculitis mechanica to describe inflammatory cutaneous lesions caused by mechanical injury on body areas which may not be prone to acne. The lesions do not present a typical clinical and histological acne vulgaris profile. $^{2}$ While acne mechanica is localized to seborrheic areas, such as the face, back and chest, folliculitis mechanica occurs anywhere a hair follicle has mechanical injury. ${ }^{2}$ Histologically, acne mechanica is identical to acne vulgaris; however, purely hyperkeratotic and 
polymorphic inflammatory lesions are seen in folliculitis mechanica. ${ }^{2}$ Clinically, acne mechanica may have chronic long-lasting flare-ups; however, flare-ups due to folliculitis mechanica quickly resolve when mechanical forces are removed. ${ }^{2}$

Even acne-free patients are experiencing first-time eruptions. 'Maskne' described by patients with a first-time eruption may represent folliculitis mechanica or acne mechanica. Furthermore, patients with a history of acne may present with folliculitis mechanica. For example, a 26-year-old violinist with a 6-year history of acne developed papulopustular and painful nodulocystic lesions below the right jawline ${ }^{2}$ and was diagnosed with folliculitis mechanica caused by pressure from the violin. $^{2}$

Proposed treatment guidelines include wearing properly fitted masks, applying noncomedogenic moisturizers to the face before wearing personal protective equipment (PPE) to lubricate the skin and reduce friction, and avoiding washing the face with hot water or irritants like ethanol, which breakdown the skin's protective barrier. For mask-induced pruritus, placing two to three layers of gauze inside the mask may help. ${ }^{4}$

As mask use increases, dermatologists may see an increase in maskne cases. Although mask-associated facial dermatoses are largely documented in healthcare workers in the literature, the general population is clearly being affected by 'maskne'. Dermatologists should counsel patients regarding proper skin hygiene: To avoid over cleansing, mild cleansers close to skin's natural $\mathrm{pH}(\mathrm{pH} 5)$, should be used in combination with non-comedogenic moisturizing creams. Exfoliation with alpha or beta hydroxyl acids can be used to decrease hyperkeratosis. ${ }^{2}$ This skincare regimen should be sufficient for patients with folliculitis mechanica. However, if 'maskne' persists despite the above regimen, lesions may represent acne mechanica rather than folliculitis mechanica. Conventional acne vulgaris treatments may then be necessary, especially if the condition is associated with severe hyperseborrhoea. ${ }^{2}$

\section{Conflict of Interest Disclosures: None}

Funding: None

Corresponding Author:

Tamar Aliya Gomolin, BSc

40 Sunshine Cottage Rd

Valhalla, NY 10595

Phone: 514-834-0995

Email: gomolin.tamar@gmail.com

\section{References:}

1. Kutlu Ö, Güneş R, Coerdt K, Metin A, Khachemoune A. The effect of the "stay-athome" policy on requests for dermatology outpatient clinic visits after the COVID-19 outbreak. Dermatol Ther. 2020:e13581.

2. Dreno B, Bettoli V, Perez M, Bouloc A, Ochsendorf F. Cutaneous lesions caused by mechanical injury. Eur J Dermatol. 2015;25(2):114-21.

3. Han C, Shi J, Chen Y, Zhang Z. Increased flare of acne caused by long-time mask wearing during COVID-19 pandemic among general population [published online ahead of print, 2020 May 29]. Dermatol Ther. 2020;e13704. doi:10.1111/dth.13704

4. Masood S, Tabassum S, Naveed S, Jalil P. COVID-19 Pandemic \& Skin Care Guidelines for Health Care Professionals. Pak J Med Sci. 2020;36(COVID19-S4):S115-S7. 